\title{
Heat pain thresholds in the oral-facial region
}

\author{
BARRY G. GREEN \\ Monell Chemical Senses Center, Philadelphia, Pennsylvania
}

\begin{abstract}
Two experiments demonstrate that the sensitivity to heat pain varies significantly within the oral-facial region. Although the chin, the vermilion border of the lip, and the tonguetip are about equally sensitive, the mucosal lip and the dorsum of the tongue produce thresholds approximately $1.5^{\circ} \mathrm{C}$ higher. The spatial heterogeneity of heat-pain thresholds is discussed in relation both to local variations in perceived warmth in the same areas and to the neural and physical variables that might underlie them.
\end{abstract}

It was recently demonstrated that the responsiveness of the oral-facial region to suprathreshold warming and cooling is heterogeneous (Green, 1984). Spatial variations are especially large for warmth, with differences in perceived warmth intensity reaching ratios of as much as $2: 1$ between the tonguetip and the dorsum of the tongue and $3: 1$ between the hairy skin of the outside of the lip and the mucosal skin of the inside of the lip. In addition, the psychophysical functions generated on those areas show little or no tendency toward convergence at the highest skin temperatures tested (ca. $42^{\circ}-44^{\circ} \mathrm{C}$ ). The latter finding provided the impetus for the present study: The absence of convergence at temperatures that produced strong sensations of warmth raised the possibility that heat-pain thresholds might be unusually high on some oral loci.

Common experience seems to support the idea that the mouth is relatively insensitive to thermal pain. Hot but drinkable coffee and tea are held in insulated containers or in cups with handles to avoid burning the hands, and cooked foods such as baked potatoes are often eaten when still too hot to handle. Although oral intake of such foods can be modified to accommodate their high temperatures (e.g., by sipping or eating small portions), one need only dip a finger briefly into a hot beverage to become suspicious of the mouth's sensitivity to potentially harmful temperatures. It was this observation that apparently motivated Margarida, Hardy, and Hammel (1962) to make the only previous measurements of the heat-pain threshold in the oral cavity. Testing only the hard palate, Margarida et al. obtained a threshold of $46.6^{\circ} \mathrm{C}$, which they judged was not significantly different from heat-pain thresholds on the rest of the body (see below). However, the absence of simultaneous threshold measurements on other loci

The author thanks Barbara Gelhard for her help in carrying out the experiments of the study and assisting with the data analysis. This research was supported in part by a grant from the National Institutes of Health (NS 20577).

The author's mailing address is Monell Chemical Senses Center, 3500 Market Street, Philadelphia, PA 19104. either inside or outside the mouth rendered this conclusion tenuous. The possibility remained that other regions of the mouth (e.g., the tongue) might be less sensitive than the palate, and that the palate itself might be found significantly less responsive than extraoral skin if additional measurements were made with the same subjects and the same stimulator.

The presence or absence of spatial variations in the heatpain threshold could have relevance to theories of the sensory coding mechanisms that underlie thermal pain. The only thorough regional survey to date of heat-pain (or "pricking pain") thresholds on external skin has indicated that sensitivity did not vary over most of the body's surface. Hardy, Wolff, and Goodell (1952) measured the threshold for pain produced by radiant heat on 32 loci and concluded "the pain threshold is approximately uniform over the body surface'" (p. 427). Hardy et al. published no statistical tests to prove this contention, but it appears from their data that only the heavily calloused heel of the foot produced a threshold $\left(53.7^{\circ} \pm 2.2^{\circ} \mathrm{C}\right)$ significantly above the mean for the rest of the body $\left(44.0^{\circ} \pm 1.1^{\circ} \mathrm{C}\right)$. $\mathrm{A}$ few other areas evinced thresholds a degree or more different from the overall mean but also produced standard deviations of similar magnitude (e.g., the lower back, $42.2^{\circ} \pm 1.4^{\circ} \mathrm{C}$ ). The apparent uniformity of sensitivity except where the epidermis is unusually thick supports the notion that heat pain is triggered by a specific population of receptors which become active at injurious or nearinjurious temperatures (Hardy, Goodell, \& Wolff, 1951').

Consistent with a relatively "fixed" pain threshold was an early observation made by Hardy, Wolff, and Goodell (1940) that the threshold for heat pain did not vary with the size of the thermal stimulus. In addition, more recent psychophysical analyses of the effect of stimulus size on perceived warmth have supplied indirect evidence that the sensory system or systems serving heat pain may not summate spatially (Stevens \& Marks, 1971; Stevens, Marks, \& Simonson, 1974). However, Greene and Hardy (1958) later modified the earlier conclusion that spatial summation was insignificant for pricking pain after 
another experiment had revealed a statistically significant difference in the threshold for a $2.5-\mathrm{cm}^{2}$ area, compared with areas of 7.8 and $16 \mathrm{~cm}^{2}$. The authors remarked that the $1.1^{\circ} \mathrm{C}$ difference in thresholds they obtained "represents a highly significant amount of summation" (p. 463). The absence of a difference in thresholds for the two larger areas in the Greene and Hardy study implies that spatial summation of heat pain may come into play only when relatively few receptors are activated, as when stimulus size is small or innervation density is low. Because oral tissue varies markedly in innervation density (Dixon, 1962; Marlow, Winkelmann, \& Gibilisco, 1965), spatial differences in pain thresholds detected inside the mouth might provide an opportunity for learning whether or not spatial integration contributes to the perception of heat pain.

The two experiments reported here demonstrated that significant differences indeed exist among heat-pain thresholds at five oral-facial sites. The first experiment revealed that the inner surface of the lip and the dorsum of the tongue are considerably less responsive to high temperatures than the vermilion lip and the chin. The second experiment showed that, unlike the dorsal-medial surface of the tongue, the tip of the tongue is reasonably sensitive to heat pain.

\section{EXPERIMENT 1}

This experiment compared the thresholds for perception of heat pain on areas of skin in the oral-facial region known to differ in responsiveness to moderate warming (Green, 1984). On the basis of the earlier data, it was hypothesized that the mucosal lip and the dorsum of the tongue would have abnormally high thresholds, whereas the vermilion lip and the hairy skin outside the mouth would have lower thresholds.

\section{Method}

Subjects. Seven young adults (five females and two males, average age $=24.4$ years) were paid to participate. All had served previously in psychophysical experiments, but none had experience making numerical judgments of thermal pain.

Apparatus. The thermal stimuli were delivered via a Peltier thermoelectric module. The module was glued to a water-circulated $\left(30^{\circ} \mathrm{C}\right)$ heat sink that was, in turn, clamped to a modified stereotaxic device to enable flexible positioning of the stimulator. The face of the module $\left(2.54 \mathrm{~cm}^{2}\right)$ was fitted with a thin piece of aluminum sheeting to provide a smooth stimulating surface, upon which a layer of silicone glue (approximately $4 \mathrm{~mm}$ thick) was applied to all but a small rectangular area $(5 \times 25 \mathrm{~mm})$ at one end of the module. The exposed rectangle constituted the stimulating thermode. Silicone glue was also applied to the sides of the module to seal it from moisture and to reduce spurious heat radiation to skin adjacent to the stimulation site. The temperature of the thermode was monitored with a microthermocouple (approximately $0.13 \mathrm{~mm}$ diam) centered on the exposed surface, its connecting wire passing beneath the insulating silicone on the remainder of the module and on to a digital electronic thermometer (SensorTech BAT-12). In addition to the thermometer readout, a continuous record of the temperature at the thermode-skin interface was made by leading the analog output of the thermometer to a chart recorder (Omega Model 585).

Temperature was controlled during the experiment by manually increasing the current flow from the power supply to the thermoelectric module at the rate necessary to cause the pen on the recorder to trace along a diagonal line drawn on the chart paper. The slope of the line was appropriate to produce a $0.1^{\circ} \mathrm{C} \mathrm{sec}-1$ increase in thermode temperature at the preselected chart speed of $6 \mathrm{~cm} \cdot \mathrm{min}^{-1}$. Controlling skin temperature by this method proved satisfactory for such a slow rate of temperature change. Only rarely did movement by the subject or misadjustment by the experimenter produce large deviations (e.g., rapid changes of $0.2^{\circ}-0.3^{\circ} \mathrm{C}$ ) from the target $\Delta \mathrm{T}$, at which time the trial was terminated and discarded.

Procedure. The subject reclined comfortably in a modified dental chair equipped with a headrest. The stimulator was suspended on a movable arm that allowed placement of the thermode against the four areas to be tested: the dorsal surface of the tongue approximately $3 \mathrm{~cm}$ from the tip, the mucosal skin of the inner wall of the lower lip, the vermilion border of the lower lip, and the middle of the chin (dorsal aspect). To stimulate the dorsum of the tongue, the subject extended the tongue as far forward as possible without discomfort. The angle of the thermode was adjusted to rest firmly against the tongue, and the edge of the silicone insulation covering the module was beveled to provide full contact of the $1.25-\mathrm{cm}^{2}$ stimulating surface. The mucosal lip was contacted by rolling the lower lip forward and lowering the thermode against its inner wall, with the thermode angled so that it held the lip in place while touching only the mucosal skin (i.e., the insulated portion of the module extended over, but did not contact, the down-turned vermilion border). Stimulation of the vermilion border was achieved with the thermode in a similar position but with the lower lip in its normal orientation. The thermode was lowered and its angle was changed to contact the upper surface of the chin, the insulated portion of the model extending over but not touching the skin of the lower part of the chin.

Thresholds were measured by use of an ascending method of limits. After the thermode was positioned on one of the skin loci, the temperature at the skin-thermode interface was brought to between $34^{\circ}$ and $37^{\circ} \mathrm{C}$, where it was stabilized. (These starting temperatures encompassed normal skin temperatures at the different test sites. Subjects reported only mild warmth or thermal neutrality immediately after contact with the thermode.) Both the exact starting temperature and the delay between thermode contact and initiation of a temperature increase were varied over trials to discourage subjects from attempting to standardize their thresholds by responding after a fixed interval on each trial.

The subject was instructed to consider the heat-pain threshold as the moment at which the thermode first became painfully hot. No attempt was made to describe "painfully hot' in qualitative terms (e.g., pricking or stinging), in part because it was questionable whether the qualitative nature of heat pain would be the same on the different kinds of skin to be tested. Emphasis was placed instead on the more functional concept of a surface becoming sufficiently hot to cause fear that continued contact at that temperature might eventually lead to burn. This criterion appears akin to Neisser's (1959) "point of spontaneous withdrawal," which he reported produced thresholds similar to but more variable than thresholds for pricking pain. (The additional variability may have come from Neisser's instructions to subjects to "keep your mind on something else" during trials in which thresholds were terminated by spontaneous withdrawal.)

The subjects signaled the onset of heat pain by pressing a hand switch to produce a mark in the temperature trace on the chart recorder. Thermode temperature was immediately reduced, and the subject was told to push the thermode away from the skin. An intertrial interval of at least 2 min was imposed to allow skin temperature to return to normal and the thermal sensation to diminish. Additional time was given if, after $2 \mathrm{~min}$, the subject reported lin- 
gering sensations of warmth or heat. Stimulation then proceeded on the same location two additional times to yield three estimates of the pain threshold per site per day. A total of 15 threshold estimates was obtained from every subject over five experimental sessions, each of which lasted about $1 \mathrm{~h}$.

\section{Results}

Figure 1 displays the combined results for all seven subjects. The datum for each locus is therefore based on 105 threshold measurements $(7 \times 15)$.

The results demonstrate that differences exist in the heat-pain threshold among oral-facial loci. A two-way ANOVA with repeated measures established that the spatial differences were significant $[F(3,18)=18.45, \mathrm{p}<$ $.001]$, as were the differences in threshold estimates obtained over the five test sessions $[F(4,24)=11.02, p<$ $.001]$.

The most sensitive loci were the vermilion lip and the chin, which had thresholds of $46.0^{\circ}$ and $46.3^{\circ} \mathrm{C}$. In contrast, thresholds on the mucosal lip and the dorsum of the tongue averaged $47.5^{\circ}$ and $47.8^{\circ} \mathrm{C}$, exceeding the thresholds on hairy and glabrous skin by an average of $1.5^{\circ} \mathrm{C}$.

Evidence of the consistency of these spatial disparities is given in Figure 2, where the means for all subjects on the 1st day of testing are compared with their means on the 5th, and final, day of testing. Although thresholds rose substantially throughout the experiment, the pattern of results across loci was unchanged. The trend toward higher thresholds with repeated testing has long been known (Neisser, 1959), and is probably due to the subjects' increasing familiarity with the experimental situation together with the growing realization that low levels of heat pain are innocuous. The instructions given to subjects in the present experiment may have invited them to delay signaling threshold as they learned that the rate of temperature change was slow and that the first appearance of heat pain was not indicative of an imminent burn.

Subjects differed substantially in their apparent sensitivity to heat pain. Thresholds on the most sensitive locus, the vermilion lip, varied from $45.6^{\circ} \mathrm{C}$ for one subject to $46.7^{\circ} \mathrm{C}$ for another. The latter subject also had the highest threshold on mucosal skin, $48.9^{\circ} \mathrm{C}$ on the tongue, whereas another subject's lingual threshold was only $46.4^{\circ} \mathrm{C}$. Regardless of these between-subject differences in absolute level, every subject had higher thresholds on mucosal skin than on hairy (chin) and glabrous (vermilion lip) skin.

\section{EXPERIMENT 2}

The higher heat pain thresholds on the mucosal lip and the dorsum of the tongue implied that the interior of the mouth might be less sensitive than the rest of the oralfacial region to high temperatures. However, because local variations in pain thresholds in Experiment 1 correlated well with local variations in perceived warmth,

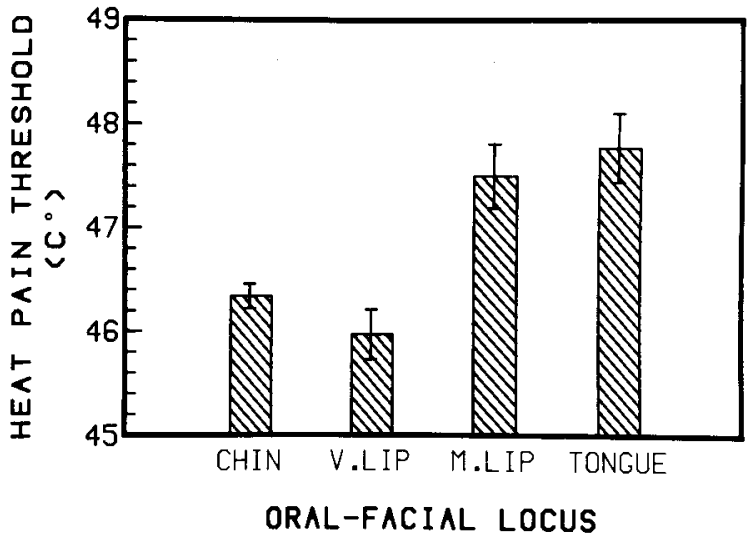

Figure 1. The mean heat-pain thresholds obtained from seven subjects over five experimental sessions. Error bars represent the standard errors of the means. (V. Lip = vermilion border of the lip; M. Lip = mucosal lip; Tongue $=$ dorsal surface $2-3 \mathrm{~cm}$ proximal to the tip).

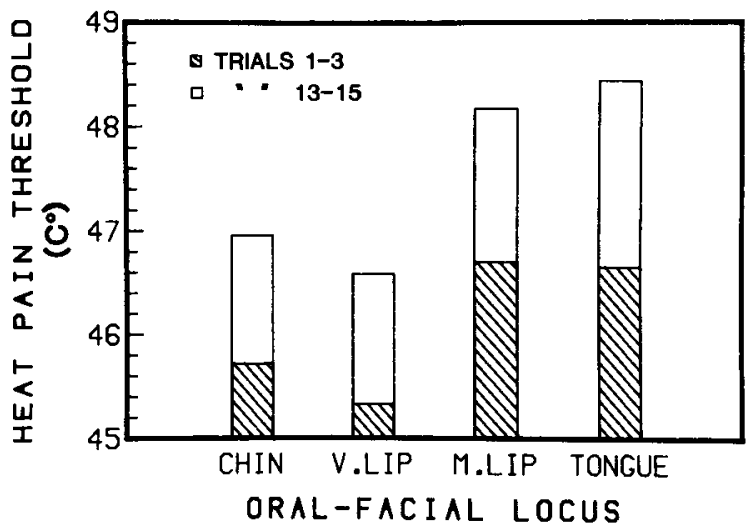

Figure 2. The mean heat pain thresholds calculated over the three trials of the first session (hatched bars) and the fifth session (empty bars) for seven subjects.

it was possible that an oral area more sensitive to warming than the mucosal lip and the dorsum of the tongue might also possess greater sensitivity to noxious temperatures. The tonguetip was considered the best location on which the test this idea, because it was found previously to be approximately twice as responsive to warming as the dorsum of the tongue (Green, 1984).

\section{Method}

Subjects. Five of the subjects of Experiment 1 (four females and one male) remained available for testing in the present experiment.

Apparatus and Procedure. The equipment and experimental procedure were basically the same as those of Experiment 1, with the exceptions that the test sites were restricted to two lingual loci and data collection was accomplished in two sessions rather than in five. The loci stimulated were the dorsal aspect of the tip of the tongue and its medial-dorsal surface about $3 \mathrm{~cm}$ proximal to the tip. Trials were run in blocks of three at each locus, with a total 
of six measurements made on each site in every session. The order of testing was counterbalanced between days so that subjects who were tested first on the tonguetip in Session 1 were tested first on the tongue dorsum in Session 2. Three subjects received stimulation on the dorsum first, and two received stimulation on the tip first.

\section{Results}

Table 1 shows that all five subjects had lower heat pain thresholds on the tip of the tongue than on the dorsum of the tongue $[\mathrm{t}(4)=8.86, \mathrm{p}<.001]$. The sensitivity of the tip exceeded the sensitivity of the dorsum by an average of nearly $2^{\circ}$, which indicates that high temperatures are sensed on the tonguetip about as effectively as on the vermilion lip and the chin. The mean threshold on the dorsal lingual surface for the same five subjects in Experiment 1 was $47.8^{\circ}, 0.7^{\circ} \mathrm{C}$ lower than their mean in the present experiment. If we assume that this difference is due primarily to a continuing rise in the threshold with experience, it is reasonable to subtract the same $0.7^{\circ} \mathrm{C}$ from the threshold for the tonguetip to arrive at an estimate for comparison with the data of Experiment 1. Doing so brings the tonguetip threshold to $45.9^{\circ} \mathrm{C}$, a value less than that for the chin and equal to the threshold for the vermilion lip. The tonguetip clearly aligns more closely with the relatively sensitive oral-facial areas than it does with the less sensitive mucosal areas. This finding eliminates skin type as the critical variable for heatpain thresholds: Mucosal skin can be as sensitive as hairy skin.

\section{GENERAL DISCUSSION}

Compared with other areas of the body (Hardy et al., 1952), the oral-facial region is unusually heterogeneous in its responsiveness to high skin temperatures. In the distance of $2-3 \mathrm{~cm}$ from vermilion lip to mucosal lip, or tonguetip to tongue dorsum, the heat-pain threshold climbs significantly higher (approximately $1.5^{\circ}-2.0^{\circ} \mathrm{C}$ ). This pattern of responsiveness parallels in a general way the variations in responsiveness to warmth in the same region. One discrepancy between warmth and pain is that warmth intensity declines from hairy facial skin to glabrous labial skin (Green, 1984, Figure 3), whereas no difference in heat pain thresholds appears between the chin and the glabrous lip. This result is not particularly surprising given

Table 1

Mean Heat Pain Thresholds for Five Subjects at Two Lingual Loci

\begin{tabular}{|c|c|c|c|}
\hline \multirow[b]{2}{*}{ Subject } & \multicolumn{2}{|c|}{ Tongue Locus } & \multirow[b]{2}{*}{ Difference } \\
\hline & Tip & Dorsum & \\
\hline 1 & 47.2 & 49.0 & 1.8 \\
\hline 2 & 45.5 & 47.1 & 1.6 \\
\hline 3 & 45.4 & 46.7 & 1.3 \\
\hline 4 & 47.6 & 50.1 & 2.5 \\
\hline 5 & 47.2 & 49.4 & 2.2 \\
\hline Mean & 46.6 & 48.5 & 1.9 \\
\hline SE & 0.5 & 0.7 & 0.2 \\
\hline
\end{tabular}

Note-All values are centigrade degrees. that warmth and heat pain are probably mediated by different kinds of receptors (e.g., Bessou \& Perl, 1969; Croze, Duclaux, \& Kenshalo, 1976; Torebjörk, 1979; Van Hees \& Gybels, 1981).

Based upon current knowledge of sensory physiological function, it is not possible to arrive at a clear understanding of why the heat-pain threshold varies across oralfacial sites. Although the neuroanatomy of the region has been well studied, the distribution of specific types of temperature-sensitive receptors is unknown. Neuroanatomical structure has not yet been related to sensory function, which makes application of the anatomical data to the present results problematic. It is possible, for example, that the increased neural density in the anterior of the tongue represents more numerous thermoreceptors and/or mechanoreceptors, but not more numerous heat nociceptors. We may speculate, however, that both the higher innervation density and the more superficial location of the nerve endings in the dorsal tonguetip compared with the dorsal-medial tongue (Dixon, 1962; Marlow et al., 1965) contribute to the lower pain threshold at the former site. Which variable contributes more depends upon the extent of spatial summation that occurs in the nociceptive system and upon the actual differences in nociceptor density between the two sites. As pointed out in the introduction, spatial summation may be significant in the heat nociceptive system only when a relatively small number of end organs are stimulated. It is plausible that some areas of the oral mucosa possess so few heat nociceptors that higher temperatures may be required to reach a threshold level of neural activity on those regions. The coincidence of significantly lower pain thresholds with significantly lower innervation densities therefore invites neurophysiological analyses of possible summation effects in the system responsive to painful levels of heat. The large spatial differences in heat-pain thresholds pose problems for psychophysical studies of summation in the oral cavity, because stimuli of different size will tend to encompass different amounts of "sensitive" and "insensitive" skin. A study is planned, however, in which the length of a metallic stimulator placed crosswise on the dorsum of the tongue will be varied systematically, with the intent of avoiding the gradient of sensitivity that runs rostrocaudally on the tongue.

Two other factors must be considered as potential determinants of heat-pain thresholds: differences in receptor type and differences in skin thickness. Variations in threshold may simply reflect activity in different types of nociceptors (i.e., ones having higher or lower thermal thresholds) at different loci. On the other hand, a logical explanation for the elevated threshold on the labial mucosa is that the epithelium is thicker there and consequently a better thermal insulator than the epithelium of glabrous or hairy skin (DiFiore, 1974; Halata \& Munger, 1983; Seto, 1963). This explanation is compromised by the absence of a difference in heat-pain threshold between the chin and the vermilion lip, which also differ in epidermal thickness (Seto, 1963). In addition, an increase in the 
moisture of tissue has been shown to improve thermal conduction (Lipkin \& Hardy, 1954) and produce stronger sensations of warmth (Green, 1984), which suggests that the thickness of the labial mucosa should be counteracted at least in part by its moistness.

The functional and behavioral implications of the present findings are more straightforward. As with perception of warm objects, perception of hot objects tends to be superior on external areas of the mouth (e.g., hairy and glabrous skin of the lip and face); the tonguetip may be the sole intra-oral site capable of sensing high temperatures as well as external skin. [Future studies will, however, investigate the thermal sensitivity of the anterior hard palate. Histological analysis (Dixon, 1962) has shown that the innervation density of the hard palate rivals that of the tonguetip, and Margarida et al. (1962) reported it had a heat-pain threshold similar to thresholds measured previously on external skin.] The sensitivity of the tonguetip makes sense behaviorally inasmuch as the tongue's mobility enables it to extend outside the mouth for use as an exploratory organ (Gibson, 1967). Such a capability probably produced environmental pressures throughout evolution favoring the development of adequate thermal and mechanical sensitivity at the tonguetip. In contrast, the interior of the mouth was probably not routinely subjected to high temperatures until the relatively recent (in evolutionary terms) use of fire to heat food. All evidence, both sensorineural (e.g., Seto, 1963) and psychophysical (e.g., Boring, 1915), points to use of the hands, lips, and tonguetip to assess the thermal suitability of potential foods before ingestion. In view of this, it is interesting to consider that most eating and drinking utensils enable us to insulate our hands, and to some extent our lips, from extremely hot or cold ingesta. Perhaps the development of such utensils was at least encouraged by the recognition that some comestibles too hot to touch nevertheless produce innocuous or even pleasing sensations in the oral cavity.

\section{REFERENCES}

Bessou, P., \& PERL, E. R. (1969). Response of cutaneous sensory units with unmyelinated fibers to noxious stimuli. Joumal of Neurophysiology, 32, 1025-1043.

Borrng, E. G. (1915). The sensations of the alimentary canal. American Journal of Psychology, 26, 1-57.

Croze, S., Duclaux, R., \& Kenshalo, D. R. (1976). The thermal sensitivity of polymodal nociceptors in monkey. Journal of Physiology (London), 263, 539-562.

DiFiore, M. S. H. (1974). Atlas of human histology. Philadelphia: Lea \& Febiger.
Dixon, A. D. (1962). The position, incidence and origin of sensory nerve terminations in oral mucous membrane. Archives of Oral Biology, 7, 39-48.

GiBsON, J. J. (1967). The mouth as an organ for laying hold of the environment. In J. F. Bosma (Ed.), Oral sensation and perception (pp. 111-136), Springfield, IL: Thomas.

GreEN, B. G. (1984). Thermal perception on lingual and labial skin. Perception \& Psychophysics, 36, 209-220.

GREENE, L. C., \& HARDY, J. D. (1958). Spatial summation of pain. Journal of Applied Physiology, 13, 457-464.

Halata, Z., \& MUNGer, B. L. (1983). The sensory innervation of primate facial skin. II. Vermilion border and mucosal lip. Brain Research Reviews, 5, 81-107.

Hardy, J. D., Goode Ll, H., \& WolfF, H. G. (1951). The influence of skin temperature upon the pain threshold as evoked by thermal radiation. Science, 114, 149-150.

HaRdy, J. D., WolfF, H. G., \& Goodell, H. (1940). Studies of pain. A new method for measuring pain threshold: Observations on spatial summation of pain. Journal of Clinical Investigation, 19, 649-657.

Hardy, J. D., WolfF, H. G. \& Goodell, H. (1952). Pricking pain threshold in different body areas. Proceedings of the Society of Experimental Biology and Medicine, 80, 425-427.

LIPKIN, M., \& HARDY, J. D. (1954). Measurement of some thermal properties of human tissues. Journal of Applied Physiology, 7, 212-217.

Margarida, R., Hardy, J. D., \& Hammel, H. T. (1962). Measurement of the thermal pain threshold of the hard palate. Journal of Applied Physiology, 17, 338-342.

Marlow, C. D., WinklemanN, R. K., \&ibilisco, J. A. (1965). General sensory innervation of the tongue. Anatomical Record, 152, 503-512.

NeISSER, U. (1959). Temperature threshold for cutaneous pain. Journal of Applied Physiology, 14, 368-372.

SETo, H. (1963). Studies on the sensory innervation (2nd ed.). Springfield, IL: Thomas.

Stevens, J. C., Marks, L. E. (1971). Spatial summation and the dynamics of warmth sensation. Perception \& Psychophysics, 9, 391-398.

Stevens, J. C., Marks, L. E., \& Simonson, D. C. (1974). Regional sensitivity and spatial summation in the warmth sense. Physiology \& Behavior, 13, 825-836.

TOREBJÖRK, H. E. (1979). Activity in c nociceptors and sensation. In D. R. Kenshalo (Ed.), Sensory functions of the skin of humans (pp. 313-326). New York: Plenum Press.

VAN Hees, J., Gybess, J. (1981). C nociceptor activity in human nerve during painful and nonpainful skin stimulation. Journal of Neurology, Neurosurgery \& Psychiatry, 44, 600-607.

\section{NOTE}

1. Hardy et al. (1951) commented that reaching the threshold for pricking pain on the lip required only about half the rate of surface heating required on the lower back $\left(80 \mathrm{mcal} / \mathrm{sec} / \mathrm{cm}^{2}\right.$ vs. $\left.150 \mathrm{mcal} / \mathrm{sec} / \mathrm{cm}^{2}\right)$. Their later publication on regional sensitivity (Hardy et al., 1952), which apparently included more extensive testing, showed the lip and lower back to have more similar thresholds $\left(200 \mathrm{mcal} / \mathrm{sec} / \mathrm{cm}^{2}\right.$ vs. 160 $\mathrm{mcal} / \mathrm{sec} / \mathrm{cm}^{2}$, estimated to produce skin temperatures of $44.1^{\circ} \pm 1.9^{\circ} \mathrm{C}$ and $42.2^{\circ} \pm 1.4^{\circ} \mathrm{C}$ ).

(Manuscript received February 18, 1985; revision accepted for publication June 26,1985 .) 